\title{
Obstacles to Option Setting: Initial Results with a Heuristic Walkthrough Method*
}

\author{
Silvia Gabrielli and Anthony Jameson \\ FBK-irst, Trento, Italy \\ \{sgabrielli, jameson\}@fbk.eu
}

\begin{abstract}
This short paper is the first step in a line of research that aims to deepen understanding of the difficulties that users often have with option setting interfaces: those parts of a system that allow the user to set parameters that influence the system's behavior and appearance. On the basis of a theoretical distinction of three things that users may fail to understand about a given option, we introduce a simple variant of the heuristic walkthrough method that helps evaluators to uncover likely obstacles. We give a quantitative and qualitative overview of the obstacles found through the application of this heuristic walkthrough to parts of four popular applications.
\end{abstract}

\section{Option Setting Interfaces as the Stepchild of Interface Design}

With regard to most aspects of mainstream graphical user interfaces and web-based systems, we have an abundance of design guidelines, along with convenient methods for inspection-based evaluation (e.g., heuristic evaluation and cognitive walkthrough). But one common part of these systems tends to remain in the shadows: those screens full of "options" (or "preferences") that each user can set to make the system work in a way that he or she will find satisfying in the long run. Everyday experience and conventional wisdom suggest that many users find it unduly difficult and/or timeconsuming to figure out suitable option settings for themselves; and several studies conducted in the 1990s (e.g., [5, 7]) already illustrated this general trend. These difficulties in turn constitute one of the motivations for interfaces that automatically adapt to their users (see, e.g., [4, 7]). What is still largely missing are constructive efforts to help designers to design better option setting interfaces, anticipating and minimizing the various obstacles that users can encounter.

An exception is found in research on privacy and security options and preferences (see, e.g., [1, 2, 3]), where concerns regarding user lack of support/guidance in option setting have been more actively debated. Our ultimate goal is to extend the relatively high level of understanding that has been achieved in this area to other types of option setting.

\footnotetext{
* The research described in this paper was conducted in the targeted research unit Prevolution (code PsychMM), which is funded by the Autonomous Province of Trento.
} 


\section{Three Types of Obstacle}

First, on a relatively theoretical level, we distinguish three types of obstacle that a user may encounter when deciding how to set a particular option:

1. Meaning unclear: The user may simply not understand the description of the option and the possible settings. For example, Mozilla Firefox offers three possible actions that the system can take when Firefox starts: Show my home page, Show a blank page, and Show my windows and tabs from last time. A (novice) user may not know whether the first alternative refers to their personal web home page or to some other "home page" (as is in fact the case).

2. Consequences unclear: Even if the user understands the meaning of all of the alternatives, they may not be aware of the consequences of each possible setting - for example, the fact that showing a blank page at startup can be much faster than the other two alternatives, especially when the user is not connected to the internet.

3. Best choice unclear: Even if the user understands the meaning and the consequences, it may be hard for them to judge which alternative will be best for them in the long run, especially if the system will be used in various different situations with which the user is not yet familiar. Where the best choice is not easy to see, the option setting interface may offer explicit advice (e.g., "recommended if you often work off-line").

Note that each of these three types of understanding does not necessarily presuppose the preceding type(s) in the list.

\section{A Variant of Heuristic Walkthrough and Its Initial Application}

To help designers and researchers think more systematically about option setting interfaces, we devised a simple variant of the heuristic walkthrough (HW) method (cf. [6]) that makes it less focused on analyzing task sequences: For each option in a given option setting screen of a given system and for each of the three aspects of clarity just discussed, our questionnaire asks the evaluator for a judgment of the likely clarity of that option for a typical user, on a scale with the values "Not clear", "Partially Clear", and "Clear". The same judgment is then also requested under the assumption that the user has read the associated online help.

Three HCI researchers (on the $\mathrm{PhD}$ and post-doctoral levels) were asked to apply the HW method sketched above, thinking of a novice user and looking at two categories of options: General and Privacy options. They analyzed a total of 79 options in 4 popular applications: Firefox 3.0.7, Skype 3.0.8.180, MS Office Outlook Web Access 2007, and the LinkedIn professional network (for registered users). They were given a worksheet that listed the 79 options and provided the coding scale. The time to complete the HW for all 4 systems was approximately 1 hour for each rater.

\section{Results}

Agreement among evaluators: For each question about each option, the "range" of the three evaluators' ratings was computed (i.e., the number of steps of the scale separating the highest and lowest ratings; possible values being 0,1 , and 2). The mean 


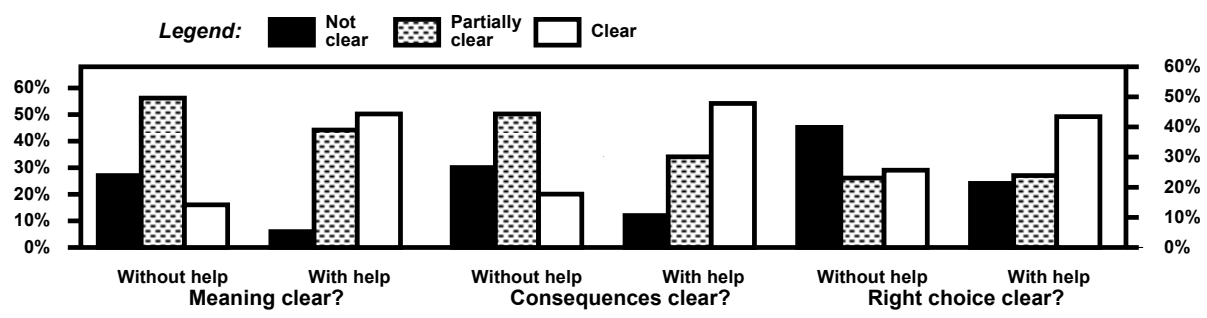

Fig. 1. Frequency distribution of the ratings for each of the three main questions of the Heuristic Walkthrough

ranges for questions concerning meaning, consequences, and overall choice were $0.91,1.02$, and 1.22 , respectively. (Since overall no systematic differences were found between the ratings of the options in the General vs. the Privacy categories, these categories will not be distinguished in the following.)

Meaning of options and alternatives: The first group of three bars in Figure 1 shows the relative frequencies of the three possible clarity ratings concerning the meaning of options, assuming that on-line help is not consulted. Only $17 \%$ of these ratings were "Clear". Often, difficulties arise when an option's description includes technical jargon. For example, in Firefox's privacy settings, a novice might not understand the term cookie in Accept cookies from third parties. But as the second group of three bars in Figure 1 shows, clarity is seen to be enhanced considerably by the online help, with "Clear" now becoming the most frequent rating. In particular, Firefox's help includes extensive information about cookies.

Consequences: As can be seen in the third group of three bars in Figure 1, the distribution of clarity ratings concerning the consequences of an option is roughly similar to that for the ratings of meaning clarity. Especially consequences that may occur only in the long term - such as those of allowing one's on-line status to be shown on the web (in Skype) were considered likely not to be appreciated. Here again, consultation of on-line help was judged to have a large impact on the recognition of relevant consequences (cf. the fourth set of three bars in Figure 1), though even with help about half of the options were considered not to be "Clear".

Choice: Not surprisingly, the evaluators saw the greatest potential for uncertainty when it came to actually making a choice about an option. For example, with the message tracking options of Outlook Webmail, it is not hard to understand the possible ways of dealing with requests for automatic responses (ask me before sending, always send, or never send). But judging which of these choices will be best in terms of the overall costs and benefits is trickier. Here again, the on-line help was seen as eliminating a good deal of the uncertainty; but still almost a quarter of the ratings are "Not clear". For example, the Outlook help about message tracking offers no advice as to the user attributes or situations that tend to be associated with particular choices. The help system writers should not necessarily be faulted: If the considerations that can influence the choice of a given setting are numerous and perhaps highly specific, there may be no alternative to letting users arrive at their decisions on their own, maybe after some trial and error. These cases remind us that the obstacles to option setting do not consist solely in a lack of provided information or in the inherent 
difficulty of technical concepts: The diversity of people and situations may be the most challenging obstacle for users and designers alike.

\section{Conclusions and Next Steps}

The initial application of our heuristic walkthrough for option setting interfaces has confirmed that it is helpful to distinguish between three types of difficulty that a user can have in deciding how to set a given option: lack of clarity about meaning, consequences, and overall choice, respectively. Although these types of clarity are interrelated in various ways, they raise different challenges for users and designers. And although all three types of clarity do not need to be present for any given option, they are all worth bearing in mind when a solution is crafted for any given option.

The fact that "help helps" (at least when it is consulted, and according to the assessments of our raters) is encouraging in some ways, but it does suggest that attention should be devoted to the question of how users can be supported in their decisions as to whether it is worthwhile for them to consult the help on a given option.

Natural next steps in this research include (a) comparing the ratings made on our heuristic walkthrough to the responses of actual users; (b) formulation and testing of guidelines for option descriptions (and the associated on-line help); and (c) expansion of the scope of the analysis to include the process of trial and error that users often rely on in addition to the information offered in the interface and the help system.

\section{References}

1. Ackerman, M.S., Cranor, L.F., Reagle, J.: Privacy in e-commerce: Examining user scenarios and privacy preferences. In: ACM Conference on Electronic Commerce, pp. 1-8 (1999)

2. Furnell, S.M.: Making security usable: Are things improving? Computers \& Security 26(6), 434-443 (2007)

3. Furnell, S.M., Jusoh, A., Katsabas, D.: The challenges of understanding and using security: A survey of end-users. Computers \& Security 25(1), 27-35 (2006)

4. Jameson, A.: Adaptive interfaces and agents. In: Sears, A., Jacko, J.A. (eds.) The humancomputer interaction handbook: Fundamentals, evolving technologies and emerging applications, 2nd edn., pp. 433-458. Erlbaum, Mahwah (2008)

5. Page, S.R., Johnsgard, T.J., Albert, U., Allen, C.D.: User customization of a word processor. In: Proceedings of CHI 1996, pp. 340-346 (1996)

6. Sears, A.: Heuristic walkthroughs: Finding the problems without the noise. International Journal of Human-Computer Interaction 9(3), 213-234 (1997)

7. Trewin, S.: Configuration agents, control and privacy. In: Proceedings of the ACM Conference on Universal Usability, Arlington, VA, pp. 9-16 (2000) 